\title{
Pre-Operative Cytological Evaluation of Thyroid Nodules According to the 2017 Bethesda System
}

\author{
Archondakis $\mathbf{S}^{*}$ \\ Department of Cytopathology, Alpha Prolipsis \\ Cytopathology Laboratories, Greece \\ *Corresponding author: Stavros Archondakis, \\ Department of Cytopathology, Alpha Prolipsis \\ Cytopathology Laboratories, 1Xenias St, Athens, 12137, \\ Greece
}

Received: December 31, 2020; Accepted: January 12, 2021; Published: January 19, 2021

\begin{abstract}
Objective: This study aims to present the experience of the implementation of the Bethesda system for reporting thyroid cytopathology in Alpha Prolipsis Medical Laboratories, a private medical laboratory located in Athens, Greece.

Methods: 617 FNAs, performed since 2017, were included in the study. Reports were issued according to The Bethesda System for Reporting Thyroid Cytopathology (TBSRTC). Aspirates were prepared with both conventional and liquid based cytology methods and were evaluated by two board certified cytopathologists. Diagnostic reproducibility and accuracy were evaluated. In 106 of these cases the cytological diagnosis was histologically confirmed.

Results: Out of the 533 cases cytologically diagnosed as benign, 7 false negative results were obtained by FNA, whereas out of the 37 cytological diagnoses of probably or definitively malignant tumors one case was found histologically to be follicular adenoma. In this trial, the diagnostic accuracy of FNA was $96.7 \%$, the specificity $94 \%$ and the sensitivity $87 \%$.
\end{abstract}

Conclusions: Our results show that FNA is a valuable examination technique in the preoperative evaluation of thyroid nodules. The integration of the 2017 Bethesda System for Reporting Thyroid Cytopathology is effective with an overall accuracy around $92 \%$.

Keywords: Thyroid; Fine-needle aspiration; Risk of malignancy; Reproducibility; Liquid-based cytology

\section{Introduction}

Thyroid nodules may be found in up to $60 \%$ of the population. The majority of thyroid nodules are benign [1,2]. Moreover, an incidental malignancy is found on histological assessment in 3-16\% of patients undergoing thyroidectomy for benign disease [1-3]. The incidence of thyroid cancer has increased worldwide in the past few decades [1-3]. FNA was recognized as a first- line diagnostic method for the evaluation of thyroid lesions in northern Europe during the period between 1950 and 1960 [2,4,5]. During the last 2 decades, its diagnostic value has been widely accepted. Nowadays, FNA consists a very useful examination due to its high accuracy in the preoperative assessment of solitary thyroid nodules, contributing to the appropriate management of the patient by decreasing the number of unnecessary thyroidectomies $[1,2,5]$. It is estimated that thyroid carcinomas consist only $1,5 \%$ of the total thyroid neoplasms and cause the 0 , $4 \%$ of cancer deaths $[1,2]$. Thyroid cancer frequency is about $0,5-10$ per 100.000 people [1,2]. Thyroid carcinomas are further subclassified in papillary, follicular, medullary, Hurthle cell, undifferentiated and metastatic. Lymphomas have also been dignosed by FNA [2,5].

Until 2007, 20-30\% of FNA reports could not be classified as either benign or malignant, partly because of factors such as the lack of a widely accepted standardized reporting format, the use of multiple, often overlapping, cytological terms in descriptive reports lacking a definite diagnosis $[1,2,6]$. A reporting system should be intronduced in order to provide definite indications concerning patient management. The proposed reporting system should be easy in everyday practice and guarantee good intra- and interobserver reproducibility for each diagnostic category [1,6,7]. The Bethesda System for Reporting Thyroid Cytopathology (TBSRTC) was introduced in 2007 to standardize terminology used in reporting thyroid cytology $[2,8]$. The six categories used are supplemented by a list of diagnostic criteria. Each diagnostic category is linked to a certain risk of malignancy [8-10]. The objective of this study was to present the 5-year experience of the implementation of the Bethesda system for reporting thyroid cytopathology in Alpha Prolipsis Cytology Laboratories, a private medical laboratory located in Athens (Greece) and to present internal quality control measures that were implemented in order to increase reliability and traceability of cytological findings and reports.

\section{Methods}

The study included patients with palpable and non- palpable thyroid nodules referred to ALPHA PROLIPSIS Cytology Laboratories during a five-year period. The laboratory is certified according to ISO 15189: 2012 and employs three board certified cytopathologists with well-documented experience in thyroid cytology. Since May 2013, Alpha Prolipsis Cytology Laboratories started reporting all thyroid FNAs using the Bethesda system and followed the guidelines in the diagnostic manual "The Bethesda System for Reporting Thyroid Cytopathology. 617 cases of thyroid FNAs were examined. The patients were directly referred to Alpha Prolipsis Cytology Laboratories. All FNAs were performed under ultrasound guidance by a consultant radiologist. All aspirations (usually three or four 
passes per lesion) were performed under ultrasound guidance with 21 -gauge needles attached to a $10-\mathrm{cm}$ syringe for suction. On-site evaluation of the specimen adequacy was performed in all cases. In case of non-diagnostic sampling, immediate repeat of the FNA was mandated. Smears were made with both conventional and liquid cytology methods and were stained with the Papanicolaou and MGG techniques. All slides were diagnosed simultaneously by two or three board certified cytopathologists. All cytopathologists used TBSRTC terminology and adhered to its diagnostic criteria. Whenever diagnostic challenging cases were encountered, the final diagnosis was made after teleconsultation with an expert colleague with well-known experience in the field. Whenever thyroidectomy was performed on the basis of FNA results or other clinical criteria, such as a multinodular lesion, nodule size or a lack of response to treatment and, in some cases, on the patient's decision because of a reluctance for periodic follow up, histological reports were methodically collected, reviewed and compared with initial cytological diagnoses. Malignancy rates for each TBSRTC category were calculated. The sensitivity and specificity of cytology for a histological diagnosis of malignancy was assessed. Statistical processing was performed with the software package IBM SPSS Statistics v.19 (IBM Corporation, Armonk, NY, USA).

\section{Results}

256 (43.6\%) male and 331 (56.4\%) female patients with a median age of 43.9 years (range, 14-82 years) and a median size of aspirated nodules of $1.6 \mathrm{~cm}$ were included in our study. A total of 587 patients underwent 617 FNAs during the study period. The incidence of each Bethesda category is summarized in (Table 1). 533 FNAs (86.3\%) were Category II (benign), 45 (7.2\%) were Category III (AUS), 4 (0.6\%) were Category IV, 28 (4.5\%) were Category V (suspicious for malignancy) and $7(1.1 \%)$ were Category VI (malignant). Of the 617 thyroid nodules from 587 patients who underwent FNA, thyroidectomy was performed to 146 (24.9\%) patients, all of whom had histopathology available for review. This comprised 75/533 (14\%) Category II cases, 32/45 (71.1\%) Category III cases, 4/4 (100\%) Category IV cases, 28/28 (100\%) Category V cases, and 7/7 (100\%) Category VI cases. The final histopathological diagnoses of cases in each category are summarized in (Table 2). Malignancy was diagnosed in 41 cases yielding an overall rate of malignancy of $6 \%$ (37/617 nodules and 41/587 patients). Of the 533 nodules diagnosed as Bethesda II (benign), 4 nodules were found to be malignant, yielding a malignancy rate of $5.3 \%(4 / 75)$ for those undergoing thyroidectomy, which represented $1.3 \%$ of the total number of Category II nodules. Of the 45 nodules diagnosed as Bethesda III (AUS/FLUS), 32 were followed up with thyroidectomy and malignancy was histologically confirmed in 5 cases with an estimated risk of malignancy of $11 \%$. There were 4 Bethesda IV nodules (Follicular neoplasm/SFN) which underwent surgery and malignancy was identified in 1 case $(25 \%)$. There were 28 Bethesda $\mathrm{V}$ nodules (suspicious for malignancy), all of which underwent surgery and $23(82.1 \%)$ were confirmed to be carcinomas, 21 papillary carcinomas and 2 medullary carcinomas. Finally, there were 7 Bethesda VI nodules (malignant), all of which underwent surgery and all $(100 \%)$ were histologically confirmed (5 papillary carcinomas, 1 medullary carcinoma and 1 primary lymphoma). The TBSRTC assigns a risk of malignancy for each diagnostic category. The estimated risk of malignancy in each TBSRTC category according to our study's results is summarized in Table 3 .

Table 1: Diagnostic Results of Our Study Population. Diagnostic Categories (2017 Bethesda System for Reporting Thyroid Cytopathology) Diagnostic category II (Benign lesions)

Nodular goiter-hyperplasia

Thyroiditis

Diagnostic category III (Atypia of undetermined significance)

Diagnostic category IV (Follicular neoplasm)

Suspicious for follicular neoplasm

Follicular neoplasm

Oncocytic neoplasm

Diagnostic category V (Suspicious for malignancy)

Suspicious for papillary carcinoma

Suspicious for medullary carcinoma

Diagnostic category VI (Malignant lesions)

Papillary carcinoma

Medullary carcinoma

Primary lymphoma

Number of cases

533

480

53

45

4

1

28

26

2

7

Table 2: Diagnostic correlation between cytology and histology reports.

\begin{tabular}{|l|c|c|c|c|c|c|c|}
\hline & I & II & III & IV & V & VI & TOTAL \\
\hline Colloid nodule & & 444 & & & & & 444 \\
\hline Graves disease & 24 & & & & & 24 \\
\hline Thyroiditis & 60 & & & & & 60 \\
\hline Adenomatoid nodule & 5 & 33 & & & & 38 \\
\hline Follicular adenoma & 2 & 1 & 1 & 2 & & 6 \\
\hline Hurthle cell adenoma & & 6 & 2 & 3 & & 11 \\
\hline Papillary carcinoma & & 4 & 5 & & 21 & 5 & 35 \\
\hline Medullary carcinoma & & & & 2 & 1 & 3 \\
\hline Follicular carcinoma & & & 1 & & & 1 \\
\hline Lymphoma & & & & & 1 & 1 \\
\hline TOTAL & & 533 & 45 & 4 & 28 & 7 & 617 \\
\hline
\end{tabular}

Table 3: Estimated risk for malignancy for each Bethesda Diagnostic Category.

\begin{tabular}{|l|c|}
\hline \multicolumn{1}{|c|}{ CYTOLOGICAL DIAGNOSIS } & Estimated risk for malignancy \\
\hline Category II & $5.30 \%$ \\
\hline Category III & $11 \%$ \\
\hline Category IV & $25 \%$ \\
\hline Category V & $82.10 \%$ \\
\hline Category VI & $100 \%$ \\
\hline
\end{tabular}

\section{Discussion}

FNA cytology is the most accurate and cost- effective method for evaluating thyroid nodules [1,2,5], It is useful for identifying features of papillary thyroid carcinoma, medullary and anaplastic thyroid carcinoma as well as other rare thyroid tumors [1,5]. Before the adoption of the TBSRTC in 2007, thyroid cytopathology reporting was following various classification schemes proposed by different scientific societies such as the Papanicolaou Society of Cytopathology and American Thyroid Association, the British Thyroid Association- 
Royal College of Physicians, the Italian Society for Anatomic Pathology and Cytopathology-International Academy of Pathology (SIAPEC-IAP). Since 2007, none of these schemes had gained international acceptance [10,11]. TBSRTC, which was adopted in 2007, is an excellent tool for the diagnosis and management of thyroid lesions [8-10]. Each of the six diagnostic categories used is associated with a sequentially increasing implied risk of malignancy that is related with specific recommendations for clinical and surgical management [8-10]. Similar to TBSRTC, all previous reporting systems were also providing categories for nondiagnostic cytology samples, benign lesions, and malignant lesions. TBSRTC proposes two distinct categories for borderline lesions: "AUS/FLUS" and "follicular neoplasm or SFN". $[8,9,10]$ The new category of AUS/ FLUS in TBSRTC is reserved for lesions not easily classified as benign, suspicious or malignant, while SFN category includes lesions suspicious of follicular carcinoma [8-10]. Unfortunately, Follicular Thyroid Carcinoma (FTC) cannot be distinguished reliably from benign follicular neoplasia on cytology. Despite the limitations of FNA cytology, secondary cytology features on thyroid FNA can raise the suspicion for FTC. These features include a hyper-cellular, monomorphic specimen with little to no colloid [5].

Based on TBSRTC, AUS/FLUS cases have a lower malignancy risk on surgical follow-up than patients with an initial diagnosis of SFN on cytology. In addition, patients with AUS/FLUS should be followed up with repeated FNA or observation, while patients with SFN should undergo at least thyroid lobectomy in order to rule out follicular carcinoma [8-10]. The introduction of AUS/FLUS category in TBSRTC classification decreased the percentage of cytologically benign FNA samples and increased the number of patients referred for thyroidectomy. The implementation of TBSRTC classification increased the number of preoperative thyroid FNAs and the number of patients undergoing thyroidectomy for indeterminate FNA results [10-12]. The frequency of each TBSRTC diagnostic category reported in our laboratory is within the ranges reported in other studies $[13,14]$. The incidence of Category III diagnosis in our study is around $11 \%$. The diagnostic criteria of AUS/FLUS category in TBSRTC include heterogeneous morphological features such as prominent population of microfollicles in an aspirate that does not fulfill the criteria for "follicular neoplasm/SFN," predominance of Hurthle cells in a sparsely cellular aspirate with scant colloid, presence of follicular cell atypia, marked cellularity of samples composed exclusively of Hurthle cells, focal features suggestive of papillary carcinoma, presence of atypical cyst lining cells, presence of follicular cells showing nuclear enlargement with prominent nucleoli, or presence of atypical lymphoid cells $[12,14]$. The diagnosis of AUS/FLUS is subjective with wide interobserver variability, but the simultaneous diagnosis by at least 2 experienced cytopathologists, as well instant teleconsultation with a very experienced cytopathologist, by means of static telecytology images transmision via passwordprotected accounts allowed excellent reproducibility and accordance with the expected ratios of AUS/FLUS diagnosis [12,13]. According to the TBSRTC management guidelines for Category III nodules, FNA should be repeated after 3-6 months $[1,2]$. In our laboratory. FNAs were repeated in 13 out of 45 cases, while thyroidectomy was decided by clinicians in 32 cases. The high number of thyroidectomies may reflect the clinicians' effort to prevent any clinical risk by an unintentional downgrading of the cytological diagnosis. The low rate of histologically diagnosed malignancies in Category III cases proves that the laboratory's quality standards are high and in that in all cases, adequate internal quality control measures were taken in order to exclude any possible misdiagnosis or unintentional error during analytical phase. In comparison to the TBSRTC, the frequency of malignancy in our study was slightly higher for Category II and Category IV, and concordant to TBSRTC in Category III, Category V, and Category VI. The slightly high risk of malignancy (5.3\%) noticed in Category II (benign) can be attributed to cases that presented incidental malignancy and is concordant with the 2015 American Thyroid Association Management Guidelines which reported 1-10 $\%$ risk of malignancy associated with benign cytological category $[1,2]$. Our study did not include any non-diagnostic cases (Category I) because all samples were adequate for cytological evaluation. On site evaluation of specimen's adequacy was proved an excellent internal quality control measure that enabled instant repeat of the FNA for additional sampling in case of non-diagnostic aspirated material. Many factors played a significant role in the satisfactory results of our study. The use of liquid based cytology techniques was proved valuable, especially in cases where molecular studies (for the detection of BRAF mutation) or special immunocytochemical stains were applied for diagnostic purposes. The use of imageguided methods, the high experience of the radiologists involved in the sampling procedure, as well, the adequate training of laboratory personnel on slide preparation and staining are secondary factors that have resulted to excellent diagnostic results.

Our laboratory is accredited since 2012 according to EN ISO 15189: 2012. According to this international quality standard, as long as the number of mistakes committed during specimen collection, preparation, and diagnostic interpretation diminishes, all monitored quality assessors continue to improve, and vice versa [15]. The laboratory is continuously monitoring factors, such as interobserver and intraobserver agreement, which play crucial role in diagnostic reproducibility. The use of static telecytology applications for teleconsultation purposes was proved an excellent alternative method for expert opinion acquisition in diagnostically challenging cases. The most common manifestation of interobserver discrepancy is upgrading of a cytological diagnosis to a definitive carcinoma diagnosis or downgrading of a suspicious cytological diagnosis to a rather benign lesion [15]. The participants cytopathologists of our laboratory have adequate experience in interpreting thyroid cytology and are continuously monitored by means of internal quality control measures, in order to improve and enhance their diagnostic capacities by all available means, such as participation in educational activities, daily discussions on scientific topics concerning the application of TBSRTC in the everyday laboratory practice. Our study demonstrated a high sensitivity and specificity of the TBSRTC, even during the first years of its implementation. Proper clinical management according to the FNA findings can still be improved in order to avoid unnecessary thyroidectomies and to ensure that TBSRTC is applied by both clinicians and cytopathologists as an indispensable interactive collaboration tool, diminishing clinical risks and ensuring the patients best interest.

\section{References}

1. Haugen BR. 2015 American Thyroid Association Management Guidelines for Adult Patients with Thyroid Nodules and Differentiated Thyroid Cancer: What is new and what has changed? Cancer. 2017; 123: 372-381. 
2. Burman KD, Wartofsky L. Clinical Practice. Thyroid Nodules. N Engl J Med 2015; 373: 2347-2356.

3. Hakala T, Kellokumpu-Lehtinen P, Kholova I, Holli K, Huhtala H, Sand J. Rising incidence of small size papillary thyroid cancers with no change in disease-specific survival in Finnish thyroid cancer patients. Scand J Surg. 2012; 101: 301-306.

4. Elisei R, Molinaro E, Agate L, Bottici V, Masserini L, Ceccarelli C, et al. Are the clinical and pathological features of differentiated thyroid carcinoma really changed over the last 35 years? Study on 4187 patients from a single Italian institution to answer this question. J Clin Endocrinol Metab. 2010; 95: 15161527.

5. Na HY, Moon JH, Choi JY, Yu HW, Jeong WJ, Kim YK, et al. Preoperative diagnostic categories of fine needle aspiration cytology for histologically proven thyroid follicular adenoma and carcinoma, and Hurthle cell adenoma and carcinoma: Analysis of cause of under- or misdiagnoses. PLoS One. 2020; 15: e0241597.

6. Nabhan F, Ringel MD. Thyroid nodules and cancer management guidelines: comparisons and controversies. Endocr Relat Cancer. 2017; 24: R13-R26.

7. Burch HB, Burman KD, Cooper DS, Hennessey JV, Vietor NO. A 2015 Survey of Clinical Practice Patterns in the Management of Thyroid Nodules. J Clin Endocrinol Metab. 2016; 101: 2853-2862.

8. Cibas ES, Ali SZ. The 2017 Bethesda System for Reporting Thyroid Cytopathology. Thyroid. 2017; 27: 1341-1346.

9. Cochand-Priollet B, Schmitt FC, Totsch M, Vielh P. The Bethesda terminology for reporting thyroid cytopathology: from theory to practice in Europe. Acta Cytol. 2011; 55: 507-511.
10. Bongiovanni M, Spitale A, Faquin WC, Mazzucchelli L, Baloch ZW. The Bethesda System for Reporting Thyroid Cytopathology: a meta-analysis. Acta Cytol. 2012; 56: 333-339.

11. Ali S, Cibas ES. The Bethesda system for reporting thyroid cytopathology. Definitions, criteria and explanatory notes. $2^{\text {nd }}$ edition. Cham (Switzerland) Springer. 2018

12. Kholova I, Ludvikova M. Thyroid atypia of undetermined significance or follicular lesion of undetermined significance: an indispensable Bethesda 2010 diagnostic category or waste garbage? Acta Cytol. 2014; 58: 319-329.

13. Paschke R, Hegedus L, Alexander E, Valcavi R, Papini E, Gharib H. Thyroid nodule guidelines: agreement, disagreement and need for future research. Nat Rev Endocrinol. 2011; 7: 354-361.

14. Straccia P, Rossi ED, Bizzarro T, Brunelli C, Cianfrini F, Damiani D, et al. A meta-analytic review of the Bethesda System for Reporting Thyroid Cytopathology: Has the rate of malignancy in indeterminate lesions been underestimated? Cancer Cytopathol. 2015; 123: 713-722.

15. Archondakis S, Georgoulakis J, Stamataki M, Anninos D, Skagias L, Panayiotides I, et al. Telecytology: a tool for quality assessment and improvement in the evaluation of thyroid fine-needle aspiration specimens. Telemed J E Health. 2009; 15: 713-717. 